\title{
Visualization of a hyaluronan network on the surface of silicone-hydrogel materials
}

This article was published in the following Dove Press journal:

Clinical Ophthalmology

28 July 2016

Number of times this article has been viewed

\section{Katarzyna A Wygladacz \\ Daniel J Hook}

Vision Care, Bausch \& Lomb

Incorporated, Rochester, NY, USA
Correspondence: Daniel J Hook Vision Care, Bausch \& Lomb Incorporated, 1400 North Goodman Street, Rochester, NY 14609, USA $\mathrm{Tel}+\mathrm{I} 5853386580$

Email daniel.hook@bausch.com
Abstract: Biotrue multipurpose solution (MPS) is a bioinspired disinfecting and conditioning solution that includes hyaluronic acid (HA) as a natural wetting agent. Previous studies demonstrated that HA sorbed from Biotrue MPS on both conventional and silicone hydrogel ( $\mathrm{SiHy}$ ) contact lens materials; an in vitro simulated-wear test validated the presence of HA on the lens surfaces for as long as 20 hours. In this study, the morphology and distribution of HA sorbed from both Biotrue and pure HA solution on SiHy contact lens surfaces was examined. Atomic force microscopy imaging was used to illustrate the topography of fresh $\mathrm{SiHy}$ contact lens materials before and after incubation with $0.1 \%(\mathrm{w} / \mathrm{v}) \mathrm{HA}$ solution. The distribution, as well as fine details of the HA network, were resolved by first staining HA with Gram's safranin, then imaging with confocal laser-scanning microscopy and differential interference-contrast microscopy. In this approach, SiHy materials take up the dye (safranin) nonspecifically, such that the resultant safranin-HA complex appears dim against the fluorescent lens background. Balafilcon A was chosen as the representative of glassy SiHy lenses that require postpolymerization plasma treatment to increase wettability. Senofilcon A and samfilcon A were chosen as representatives of SiHy materials fabricated with an internal wetting agent. A confluent and dim HA-safranin network was observed adhered to balafilcon A, senofilcon $A$, and samfilcon A lens surfaces incubated with either $0.1 \%(w / v)$ HA solution or Biotrue MPS. Therefore, the conditioning function provided by Biotrue MPS may be in part explained by the presence of the HA humectant layer that readily sorbs on the various types of $\mathrm{SiHy}$ contact lens materials.

Keywords: contact lens, hyaluronan, MPS, AFM, CLSM, DIC microscopy

\section{Introduction}

Contact lens-surface hydration and wettability are important factors affecting both patient comfort and satisfaction. Many commercially available contact lens materials are inherently wettable as manufactured, but lose wettability after being worn on the eye for a few hours, due to lens dehydration and sorption of hydrophobic tear-film components. A traditional approach used to maintain wettability is the use of surfactants in multipurpose solution (MPS), eyedrops, and packaging solutions. Surfactants reduce solution surface tension and help moisture distribute more uniformly across the lens. ${ }^{1}$ Nonionic surfactants, such as Tetronic 1107 and 1304, in lens-care solutions have proven effective at delaying the loss of lens wettability. ${ }^{2,3}$ Another approach used to maintain lens water and improve tear confluence across a lens surface is to include in lens-care solutions a well-chosen humectant biomolecule with affinity for the lens material. These include hydroxypropyl methylcellulose (eg, Complete MoisturePlus MPS; Abbott Medical Optics Inc, Santa Ana, CA, USA), ${ }^{4}$ carboxymethyl cellulose (eg, Refresh Optive lubricating and rewetting drops; Allergan, Irvine, CA, USA), ${ }^{5}$ 
and hyaluronic acid (HA; eg, Biotrue MPS; Bausch \& Lomb Incorporated, Rochester, NY, USA). ${ }^{6-8}$

HA, a biopolymer ubiquitously present in human and animal tissues, has found broad application in medicine and biotechnology. ${ }^{9}$ Due to its unique set of biological and physical properties, such as viscoelasticity, ${ }^{9}$ hydrophilicity, ${ }^{10,11}$ and lubricity, ${ }^{10,12}$ as well as its cell-regulating function, ${ }^{13} \mathrm{HA}$ has been used in numerous ophthalmic products, ranging from simple eyedrops to the viscoelastic used in cataract surgery. ${ }^{13,14} \mathrm{HA}$ is included as a component in some contact lens-packaging solutions (eg, St Shine Optical, New Taipei City, Taiwan) ${ }^{15}$ but to our knowledge the effect of the HA in these products upon lens performance has not been studied. HA is also included as a component of some contact lens MPS (eg, Biotrue and Simply One; Contopharma, Interlaken, Switzerland). However, the molecular weight (MW) of the HA varies greatly between products, which can dramatically affect its interaction with lens materials. ${ }^{16}$ For example, incorporation of cross-linked $35 \mathrm{kDa}$ and 169 kDa HA into a poly(2-hydroxyethyl methacrylate) hydrogel lowered water-contact angle and reduced in vitro sorption of lysozyme and albumin from artificial tear fluid. ${ }^{14}$ In contrast, releasable, non-cross-linked $35 \mathrm{kDa}$ HA had no effect upon contact angle after 30 minutes, while $169 \mathrm{kDa}$ HA lowered contact angle over the 4-hour experiment, suggesting that free, low-MW HA did not remain at the hydrogel surface.

Quantitative chemical methods described in the literature to measure HA concentration include normal phase, reverse-phase ion-pair, and anion-exchange chromatography utilizing ultraviolet absorption and chemiluminescence, ${ }^{17}$ high-performance liquid chromatography (HPLC) with fluorometric detection, ${ }^{17}$ and surface-enhanced Raman spectroscopy. ${ }^{18,19}$ Qualitative methods to image HA in tissues or on surfaces are primarily microscopy-based, notably optical and confocal microscopies. ${ }^{20-22}$ Because HA is a multinegatively charged biopolymer, it binds cationic dyes, among them alcian blue (AB) $)^{22,23}$ and Stains-All (E9379; Sigma-Aldrich Co, St Louis, MO, USA). ${ }^{24}$ To our knowledge, these have been used to determine HA concentration in aqueous solution, ${ }^{25}$ but not to visualize HA on silicone hydrogel ( $\mathrm{SiHy}$ ) contact lens surfaces.

In this study, HA sorption on three commercially available SiHy contact lens materials (inherently wettable senofilcon A and samfilcon A, as well as plasma-treated balafilcon A) was assessed. Balafilcon A, the oldest of the three lenses, was chosen as a representative "glassy" $\mathrm{SiHy}$ that requires postpolymerization plasma treatment to increase wettability. Senofilcon A and samfilcon A were chosen as representative of SiHy materials fabricated with an internal wetting agent. Both lenses were tested, because they incorporate the same wetting agent, but by different manufacturing methods. Atomic force microscopy (AFM) imaging, confocal laser-scanning microscopy (CLSM), and differential interference contrast (DIC) microscopy were utilized to characterize morphologies of the investigated contact lens surfaces, both as manufactured (fresh) and after incubation with either $0.1 \%(\mathrm{w} / \mathrm{v}) \mathrm{HA}$ or Biotrue MPS solutions. In pilot studies, the $0.1 \%(\mathrm{w} / \mathrm{v}) \mathrm{HA}$-solution concentration that is used in some contact lens comfort drops proved optimal for achieving a readily stained HA network that was well imaged by AFM. The approach described herein provides a simple and direct HA visualization method that can be easily applied to evaluate HA interaction with hydrogel materials.

\section{Materials and methods Contact lenses and HA solutions}

The purpose of this study was to investigate the interaction of HA sorbed from Biotrue MPS on three commercially available SiHy contact lens materials, balafilcon $\mathrm{A},{ }^{26}$ samfilcon $\mathrm{A},{ }^{27,28}$ and senofilcon $\mathrm{A}^{29,30}$ (Table 1). Balafilcon A (PureVision) is an FDA class ${ }^{31} \mathrm{~V}-\mathrm{A}$, low-water-content (36\%) SiHy material that requires postpolymerization plasma treatment to increase lens-surface wettability. It is fabricated from a vinyl carbamate derivative of 3-(tris[trimethylsilyloxy]silyl)propyl methacrylate, $N$-vinylpyrrolidone, a siloxane cross-linker, and a vinyl alanine wetting monomer. ${ }^{26}$ Samfilcon A (Ultra) is an FDA class $^{31} \mathrm{~V}-\mathrm{C}$, inherently wettable, low-water-content (46\%) $\mathrm{SiHy}$ material fabricated from a proprietary siloxane copolymer and $\mathrm{N}$-vinylpyrrolidone monomer. ${ }^{27,28}$ Senofilcon A (Acuvue Oasys) is an FDA class ${ }^{31} \mathrm{~V}_{-} \mathrm{C}_{\mathrm{r}}$, inherently wettable, low-water-content (38\%) SiHy material fabricated from monofunctional polydimethylsiloxane, $\mathrm{N}, \mathrm{N}$-dimethylacrylamide, 2-hydroxyethyl methacrylate (HEMA), siloxane macromer, tetraethylene glycol dimethacrylate, and polyvinylpyrrolidone (PVP) internal wetting agent. ${ }^{29,30}$

All investigated hydrogels were incubated either with $0.1 \%(w / v)$ HA solution (MW 1,800 kDa) or Biotrue MPS.

Table I Water content of lenses investigated

\begin{tabular}{lllll}
\hline Material & Water content & ${\text { FDA } \text { class }^{31}}^{31}$ & Brand name & Manufacturer \\
\hline Balafilcon $\mathrm{A}^{26}$ & $36 \%$ & V-A & PureVision & Bausch \& Lomb Incorporated (Rochester, NY, USA) \\
Samfilcon $\mathrm{A}^{27,28}$ & $46 \%$ & V-C & Ultra & Bausch \& Lomb Incorporated (Rochester, NY, USA) \\
Senofilcon $\mathrm{A}^{29,30}$ & $38 \%$ & V-C & Acuvue Oasys & Johnson \& Johnson Vision Care (Jacksonville, FL, USA) \\
\hline
\end{tabular}


HA solution was prepared as follows. HA powder was weighed on an XS105 analytical balance (Mettler Toledo, Columbus, OH, USA). The powder was transferred from the weighing dish to a sterile plastic bottle. HPLC-grade water was added to achieve a final HA concentration of $0.1 \%(\mathrm{w} / \mathrm{v})$. The HA-water mixture was stored at room temperature for 24 hours, after which the bottle was gently shaken 20 times to ensure solution homogeneity. Biotrue is a sterile, isotonic solution containing high-MW HA (MW 1.4 MDa; concentration $0.01 \%$ [w/w] $),{ }^{16}$ sulfobetaine zwitterionic detergent $(<0.1 \%)$ and Tetronic $1107(<5 \%)$ surfactant, boric acid $(<1 \%)$, sodium borate $(<1 \%)$, edetate disodium $(<1 \%)$, sodium chloride $(<1 \%)$, and a dual-disinfection system of polyaminopropyl biguanide $0.00013 \%(\mathrm{w} / \mathrm{w})$ and polyquaternium $0.0001 \%$. ${ }^{8,32}$ The synergistic combination of sulfobetaine detergent, Tetronic 1107 poloxamine surfactant, and HA mucopolysaccharide increases lens-surface wettability. Tetronic 1107 associates with contact lens surfaces, and is retained for at least 8 hours in vitro. ${ }^{2} \mathrm{HA}$, the eye's natural lubricant, ${ }^{13}$ binds up to 1,000 times its weight in water, ${ }^{33}$ and retains moisture at the lens surface. Similar to Tetronic 1107, HA associates with lens surfaces, and is retained by a number of different lens materials for up to 20 hours in vitro. ${ }^{6,7}$

Lenses were received in their respective blister packages containing buffered saline solution with $0.005 \%$ methyl ether cellulose (senofilcon A), ${ }^{29}$ borate-buffered saline (balafilcon A), ${ }^{26}$ and borate-buffered saline with poloxamine (samfilcon A). ${ }^{27}$ To effect HA sorption, lenses were removed from their respective blister packages and placed in $\mathrm{ReNu}$ leakproof lens cases (Bausch \& Lomb Incorporated), each well filled with $4 \mathrm{~mL}$ of Biotrue MPS. Lenses were incubated overnight as per the Biotrue MPS package insert to mimic the recommended lens-cleaning regimen. An identical set of lenses was incubated with $4 \mathrm{~mL}$ per well of freshly prepared $0.1 \%$ (w/v) HA solution. Samples were not rinsed before incubation with the HA solutions, because preliminary experiments showed no difference in HA sorption between lenses that were first rinsed and lenses placed into solution directly from their respective blister packages.

\section{Confocal laser-scanning microscopy imaging}

Following incubation with HA or Biotrue MPS solution, lenses were subsequently transferred to ReNu leakproof lens cases containing 3 mL Gram's safranin solution (94635; Sigma-Aldrich). After 3 minutes incubation with the solution, stained samples were rinsed gently with HPLC-grade water for 3 minutes to remove unbound dye. Stained lenses were assembled on glass microscope coverslips (Corning Inc, Corning, NY, USA), dried 24 hours or more in a nitrogen box, and imaged using inverted CLSM (FV1000; Olympus, Tokyo, Japan) equipped with DIC imaging. Individual DIC and XY (2-D) confocal images were recorded using a $4 \times$, $10 \times$, or $20 \times$ air objective, and suitable confocal magnification as necessary.

\section{Imaging of large lens areas}

DIC and 2-D confocal images were captured across the sample with a lower-magnification microscope objective $(10 \times)$. Individual images were merged using fiducial marks, defects, dust, or remaining stain particles serving as marks to guide the stitching of the master image generated using Adobe Photoshop CS5, which showed the HA-network distribution over a large lens area. Subsequently, $10 \mu \mathrm{m}$ beads (Spherotech Inc, Lake Forest, IL, USA) were randomly deposited on the surface of the stained HA network. Again, DIC and $\mathrm{XY}$ confocal images were captured across the sample with a $10 \times$ microscopy objective and stitched together into a master image. Random DIC and CLSM images showing the details of the stained HA were recorded with $20 \times$ microscope objective. The images were then localized on the master image based upon the bead distribution.

\section{Atomic force microscopy imaging}

AFM (Dimension Icon; Bruker, Billerica, MA, USA) was used to characterize the morphology of the contact lens surfaces in the dry state. The lenses were removed from their blister packages and rinsed overnight in HPLC-grade water to remove residual packaging solution. Rinsed samples were assembled on custom-made holders, dried for 24 hours or more in a nitrogen box, and characterized. The images were captured using etched Si AFM tips (Bruker) and soft-tapping mode. Tuning of the tips was done automatically through instrument software (NanoScope version $8.15)$. Images of $10 \times 10 \mu \mathrm{m}$ or $40 \times 40 \mu \mathrm{m}$ scan area were recorded. The root-mean-square (RMS) surface roughness was calculated as the standard deviation of all the height values within an image area using NanoScope Analysis version 1.40 image-processing software. The scan rate was adjusted depending upon the sample, and was lower than $1.2 \mathrm{~Hz}$.

The SiHy materials were also characterized by AFM after incubation with $0.1 \%$ (w/v) HA solution. Following incubation, lenses were gently rinsed for 3 minutes and assembled on appropriate holders. After drying for 24 hours or more in a nitrogen box, samples were imaged using soft tapping in air mode. 


\section{Results and discussion}

HA sorbed on contact lenses may increase lens performance by maintaining lens wettability. ${ }^{6}$ Sorption of HA on SiHy lenses can be chemical or physicochemical in nature. It can be effected by chemical attraction between appropriate functional groups of HA and functional groups on/in a hydrogel. Suitable (eg, porous or rough) surface morphology may also provide a mechanism for the HA biopolymer to anchor onto the polymeric material. Senofilcon A and samfilcon A materials, which are both inherently wettable but differ in water content, surface morphology, and surface chemistry, and plasma-treated balafilcon A were selected as representative SiHy materials for evaluation.

\section{AFM imaging}

Topographical images of dry SiHy contact lenses at two magnifications, as well as images after incubation of the lenses with $0.1 \%(\mathrm{w} / \mathrm{v})$ HA solution, are shown in Figure 1. All three materials exhibited a smooth surface (RMS $5.4 \pm 0.4 \mathrm{~nm}$, $2.2 \pm 0.3 \mathrm{~nm}$, and $2.5 \pm 0.4 \mathrm{~nm}$ for balafilcon A [Figure 1, $\mathrm{A}$ and $\mathrm{B}$ ], senofilcon A [Figure 1, D and E], and samfilcon A [Figure $1, \mathrm{G}$ and $\mathrm{H}$ ], respectively, based upon $10 \times 10 \mu \mathrm{m}$ image size). Nanometer-scale, pit-like features were observed on all polymers. Pits on balafilcon A were $25.6 \pm 7.9 \mathrm{~nm}$ deep. The features seen on senofilcon A were denser, but not as deep $(6 \pm 1.5 \mathrm{~nm})$ as and similar in depth to those on samfilcon A $(4.5 \pm 1.5 \mathrm{~nm})$. The average pore diameter on
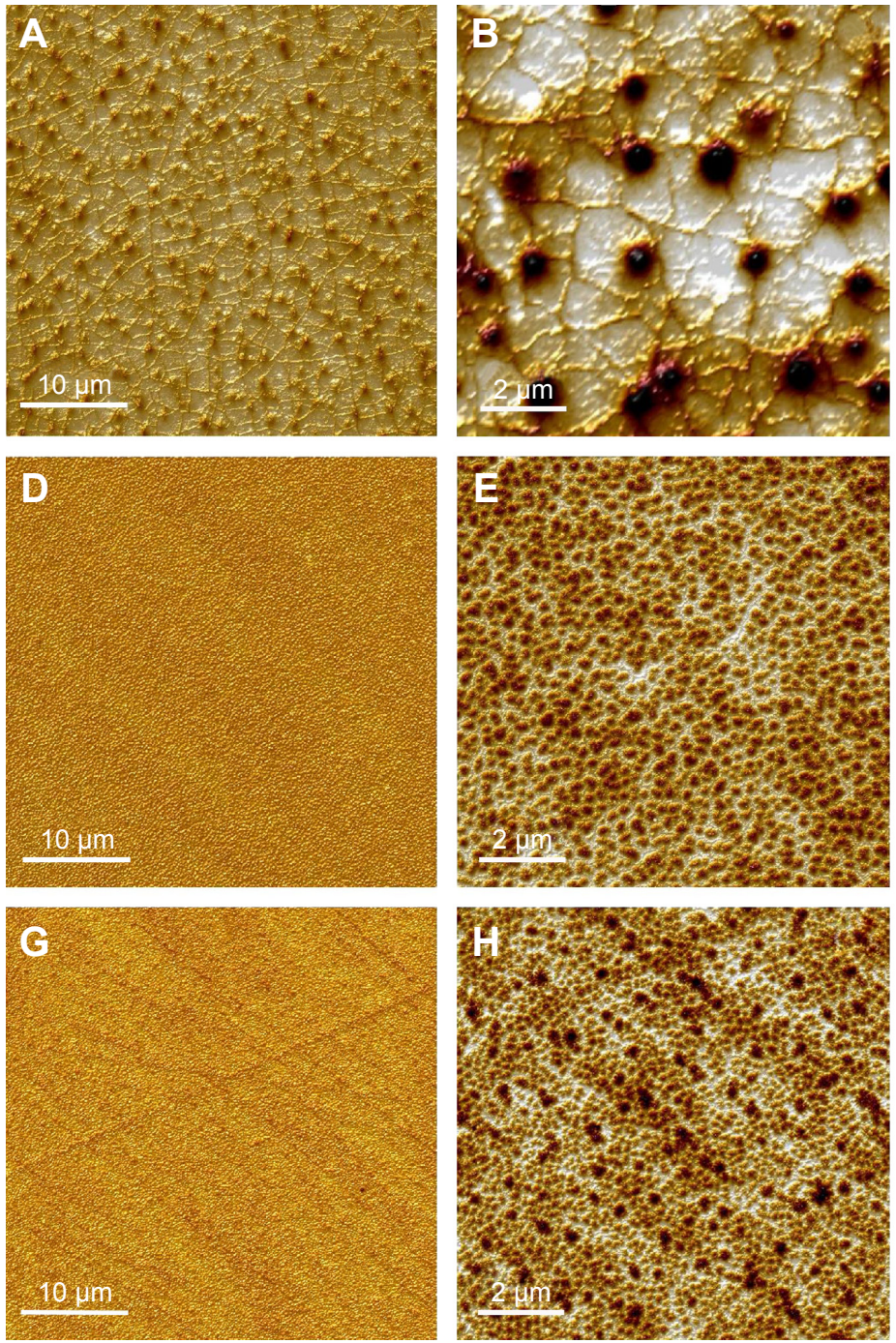
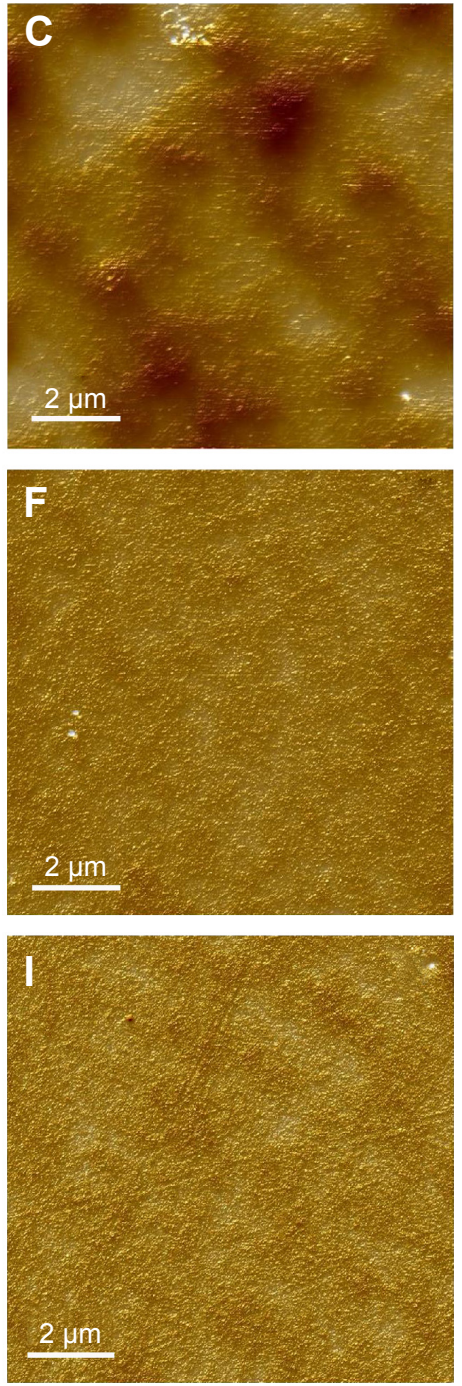

Figure I AFM topographical images.

Notes: Dry balafilcon A (A, B), senofilcon A (D, E), and samfilcon A (G, H) SiHy lenses at small $(10 \times 10 \mu \mathrm{m})$ and large $(40 \times 40 \mu \mathrm{m})$ scan size, respectively; images after overnight incubation with $0.1 \%,(w / v)$, HA solution of balafilcon $A(C)$, senofilcon $A(\mathbf{F})$, and samfilcon $A$ (I) SiHy lenses at small scan size. Sorbed HA levels the surface morphology observed on fresh lenses.

Abbreviations: AFM, atomic force microscopy; SiHy, silicone hydrogel; HA, hyaluronic acid. 
senofilcon A $(230 \pm 54.7 \mathrm{~nm})$ was similar to that on samfilcon A (189.2 $\pm 71.7 \mathrm{~nm})$, while balafilcon A showed a unique structure. After incubation with $0.1 \%(\mathrm{w} / \mathrm{v})$ HA solution, considerable diminishing of previously observed pits and other features was observed for all three hydrogels. Further, RMS surface roughness decreased to $1.9 \pm 0.6 \mathrm{~nm}, 0.7 \pm 0.4 \mathrm{~nm}$, and $0.6 \pm 0.1 \mathrm{~nm}$ for balafilcon A (Figure 1C), senofilcon A (Figure 1F), and samfilcon A (Figure 1I), respectively.

AFM section analysis of balafilcon A (Figure 2) confirmed that the sorbed HA reduced the pore/pit size, and that HA filled the pits and valleys present on the lens surface, significantly reducing their depth $(3.7 \pm 1.4 \mathrm{~nm})$. The island- and valley-like features observed on images of the fresh lens were no longer detected. HA packed the pores and smoothed the surface. Uniform coverage of the lenses with HA was observed, based upon imaging of ten randomly chosen areas.

AFM has been used previously to characterize the surfaces of various contact lenses. González-Méijome et al reported surface roughness of balafilcon A of 8.3-19.11 nm, depending upon the area analyzed. ${ }^{34}$ Lira et al similarly reported surface roughness of $9.5 \pm 0.7 \mathrm{~nm}$ over an area of $100 \mu \mathrm{m}^{2}$, compared with $13.38 \mathrm{~nm}$ from the previously mentioned study and 5.4 $\pm 0.4 \mathrm{~nm}$ in this study. ${ }^{35}$ Teichroeb et al reported surface roughness of 3.01-5.24 $\pm 0.2 \mathrm{~nm}$ for balafilcon A and 0.9-1.86 $\pm 0.2 \mathrm{~nm}$ for senofilcon A. ${ }^{36}$ They described balafilcon A as having an "island-like" structure, and senofilcon A as having a "sponge-like" surface topography.
While images of HA sorbed on contact lenses are few and not well characterized in the literature, contact lenses with both elutable and covalently bound HA have been described. Ali and Byrne used molecular imprinting principles to design lenses with high HA affinity that eluted therapeutic amounts of HA over at least 1 full day of lens wear. ${ }^{37}$ However, imaging of the HA and the lens was beyond the scope of the study. Sheardown et al studied a number of HA-containing lensmaterial variants. ${ }^{14,38,39}$ Van Beek et al used AFM to show that cross-linking relatively low-MW HA into poly-HEMA-based hydrogels increased surface roughness. ${ }^{14}$ Although similarmodel SiHy lenses with HA proved to reduce the sorption of lysozyme on lenses, HA at the lens surface was characterized by Fourier-transform infrared spectroscopy-attenuated total reflectance and X-ray photoelectron spectroscopy. ${ }^{38}$ Weeks et al also reported extended delivery (3-7 weeks) of HA from lenses into which HA was incorporated during synthesis, but did not report further surface characterization. ${ }^{39}$ Bettuelli et al used AFM to image an HA-containing contact lens (St Shine Optical) and reported surface roughness of approximately 2-3 nm over a $10 \times 10 \mu \mathrm{m}$ area, ${ }^{40}$ similar to our finding with HA sorbed on balafilcon A. However, the roughness of the analogous HA-free lens was not reported, and our HA loading procedure is different, making the comparison interesting but of unknown validity. Tavazzi et al used AFM and scanning electron microscopy (SEM) to image a SiHy lens (Safilens, Italy) before and after sorption of fluorescently labeled, $10^{3} \mathrm{kDa} H A$. Pore size decreased after incubation with the HA solution, suggesting uptake by the lens. ${ }^{41}$ Further, CLSM

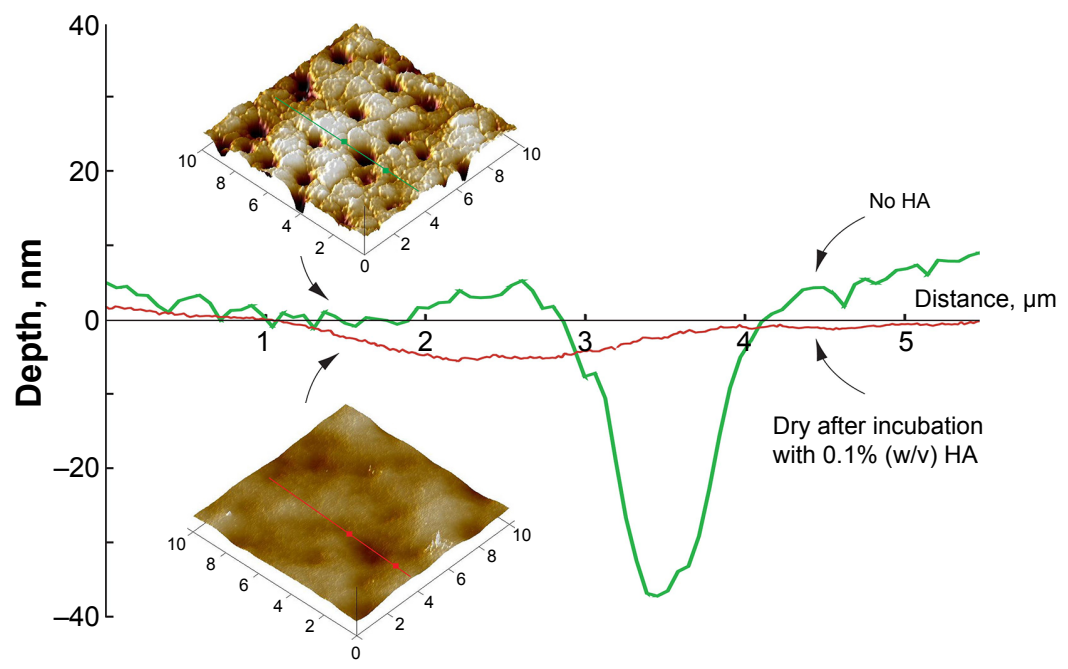

Figure 2 AFM section analysis of balafilcon A before and after overnight incubation with $0.1 \%(w / v)$ HA solution.

Notes: Samples were characterized in a dry state by soft tapping in air. Sorbed HA uniformly covered the surfaces of the hydrogels, filling the pits to decrease pit depth and decreasing surface roughness.

Abbreviations: AFM, atomic force microscopy; HA, hyaluronic acid. 
revealed discrete fluorescein emission originating 5-10 $\mu \mathrm{m}$ beneath the surface; the spacing between these points of emission was roughly the same as the spacing between the pores observed in the SEM images.

\section{CLSM and DIC imaging}

While AFM provides excellent resolution, it is not suitable for imaging of larger areas or rough samples. HA localization and its distribution can be better illustrated by using an appropriate microscopy technique. Fluorescence-based techniques are of particular interest, due to their inherent ultralow parts-pertrillion detection limit. ${ }^{42,43}$ While HA is nonfluorescent, it forms stable complexes with various cationic dyes, ${ }^{24}$ such as toluidine blue (TB), ${ }^{44}$ safranin,,${ }^{45}$ and $\mathrm{AB} .{ }^{22}$ TB metachromatically stains HA red, with maximum emission at $660 \mathrm{~nm}$ when excited at $595 \mathrm{~nm}$. Blumenkrantz reported that HA-TB products may be of various morphologies, such as filamentous or granular. ${ }^{44} \mathrm{AB}$, a derivative of a copper phthalocyanine, stains mucopolysaccharides and glycosaminoglycans. ${ }^{22}$ Safranin $(530 / 590 \mathrm{~nm} \text { excitation/emission wavelength })^{46}$ is a biological Gram stain typically used for the detection of cartilage, mucin, and mast cells. However, there have been few reports in the literature describing interactions of safranin with HA samples other than biological.
$\mathrm{TB}$ and $\mathrm{AB}$ staining of $\mathrm{HA}$ sorbed on lenses proved challenging, with $\mathrm{AB}$ the more problematic. Commercial $\mathrm{AB}$ stain is received as a saturated solution of the fluorophore. The crystals of the stain components adhered strongly to the lens materials and were difficult to rinse, thus interfering with the HA-visualization method. In contrast, safranin stain was found more suitable for imaging HA sorbed on SiHy lenses. To determine how safranin interacts with SiHy materials, fresh lenses were first incubated for 3 minutes with safranin solution. Nonspecific interaction of the dye with all lens materials was evident, and manifested as brightly fluorescing polymer (image not shown). CLSM images of SiHy materials incubated overnight with $0.1 \%$ $(\mathrm{w} / \mathrm{v})$ HA solution and stained with safranin are shown in Figure 3. Sorbed HA is readily observed on all three hydrogel surfaces as a dim film against the bright background. The morphology of the HA network was similar on all substrates, but HA density appeared greater on samfilcon A (Figure 3C) than on balafilcon A (Figure 3A) or senofilcon $\mathrm{A}$ (Figure $3 \mathrm{~B}$ ). This may reflect stronger attraction of $\mathrm{HA}$ to this hydrogel, because of its greater concentration of PVP in the lens formulation. Like samfilcon A, senofilcon A is also inherently wettable, but it is fabricated with less PVP compared to samfilcon $\mathrm{A} .{ }^{28}$ The morphology of sorbed HA
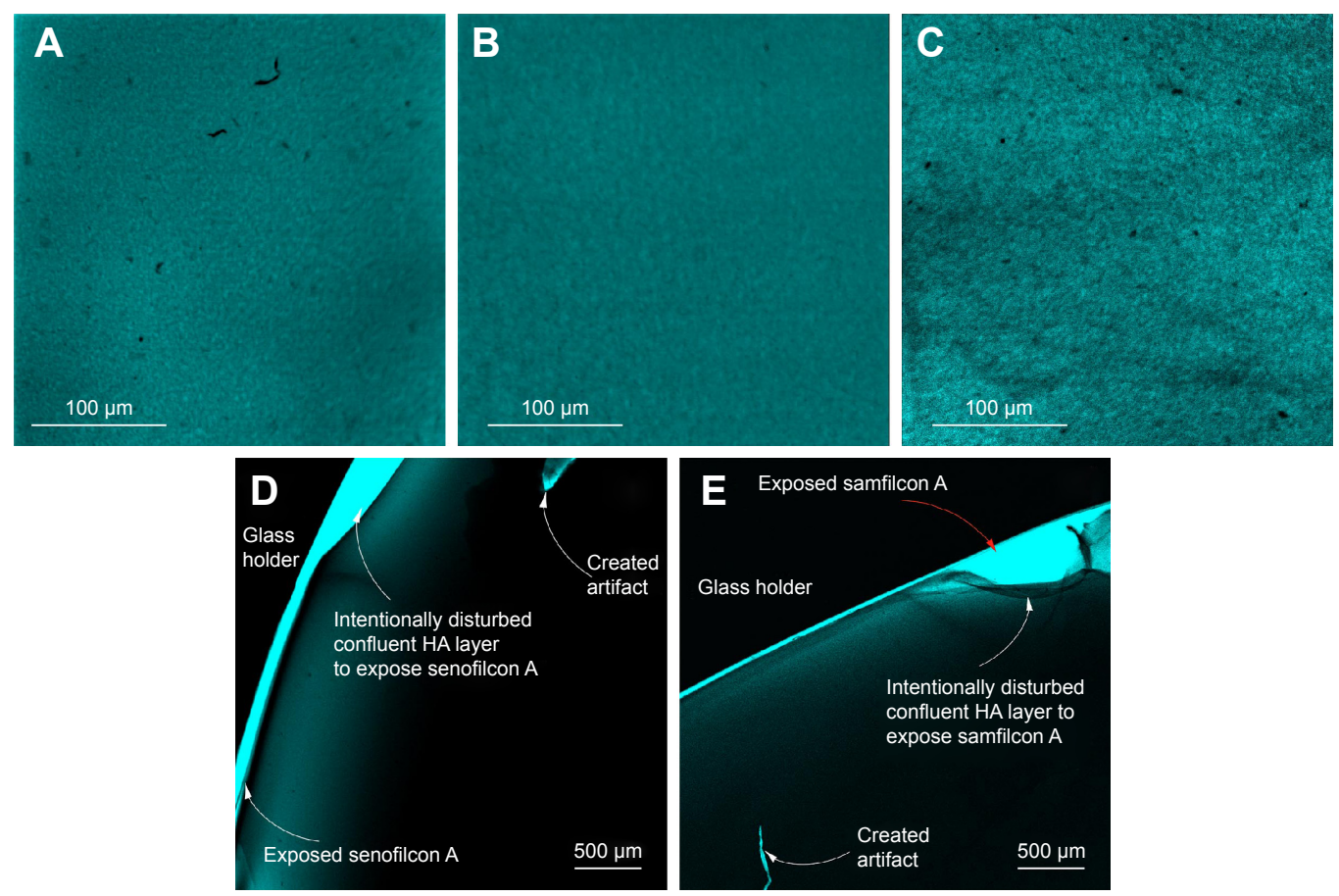

Figure 3 CLSM images.

Notes: Balafilcon A (A), senofilcon A (B), and samfilcon A (C) incubated overnight with $0.1 \%(w / v) H A$ solution, then stained with safranin; images of senofilcon $A(\mathbf{D})$, and samfilcon $A(E)$ after scratching the sorbed HA layer with forceps. HA on balafilcon A could not be imaged by this method, because the stained lens did not sufficiently adhere to the glass slide. A-C Captured using a $20 \times$ microscope objective and $2 \times$ confocal magnification; D-E were captured using a $4 \times$ microscope objective. Abbreviations: CLSM, confocal laser-scanning microscopy; HA, hyaluronic acid. 
in CLSM images resembled that observed in AFM images (Figure 1).

Imaging of larger lens areas was limited to the objective magnification available on CLSM; $3.2 \times 3.2 \mathrm{~mm}$ images were captured to demonstrate uniform coverage of HA on the SiHy materials. To validate that the observed dim films on the surfaces of the samples were the HA network stained with safranin, the films were scratched gently with tweezers. While the balafilcon A lens readily delaminated from the glass holder, the senofilcon A (Figure 3D) and samfilcon A (Figure 3E) lenses remained adhered, and the HA film tore at the site of the scratch, revealing the HA film, the defects, and the fluorescent substrate. The glass holder was not optically active, and thus appears black in the images.

The CLSM method was subsequently used to verify transfer of HA to the lens surfaces from Biotrue MPS. In control experiments, Biotrue MPS components other than HA did not interact with the stain (data not shown). CLSM images of the SiHy contact lenses after overnight incubation with Biotrue MPS and staining with safranin are displayed in Figure 4. A dim, relatively less fluorescent HA network similar to that observed on lenses incubated with HA solution (Figure 3) was evident. However, the density of HA sorbed from Biotrue MPS was less than that of HA sorbed from pure solution, due to the different HA concentrations in the two solutions. While similar HA morphology, network structure, and network distribution was observed on balafilcon A (Figure 4A) and senofilcon A materials (Figure 4B), HA density appeared greater on samfilcon A (Figure 4C). Scratched HA films behaved similarly to those adhered from HA solution, with balafilcon A delaminating, and senofilcon A (Figure 4D) and samfilcon A (Figure 4E) remaining adhered. Therefore, the HA biopolymer appeared to effectively sorb from both HA solution and Biotrue MPS on all SiHy materials evaluated.

To our knowledge, this is the first study to report CLSM images of unlabeled HA on contact lens surfaces. However, several studies have used fluorescein-labeled HA (F-HA) to image interactions between HA and contact lenses. Weeks et al loaded F-HA into conventional lens materials, and used CLSM to determine the distribution of HA within the hydrogel. ${ }^{47}$ They used Z-stack CLSM to determine that the depth of penetration of HA into the lens was a function of HA MW. Singh et al similarly used F-HA to image sorption of HA on PureVision lenses that had been modified with an HA-binding ligand. ${ }^{48}$ One potential disadvantage of this technique is that F-HA and HA may interact differently with lenses, while the stain used in our study interacts with HA already sorbed on the lens.
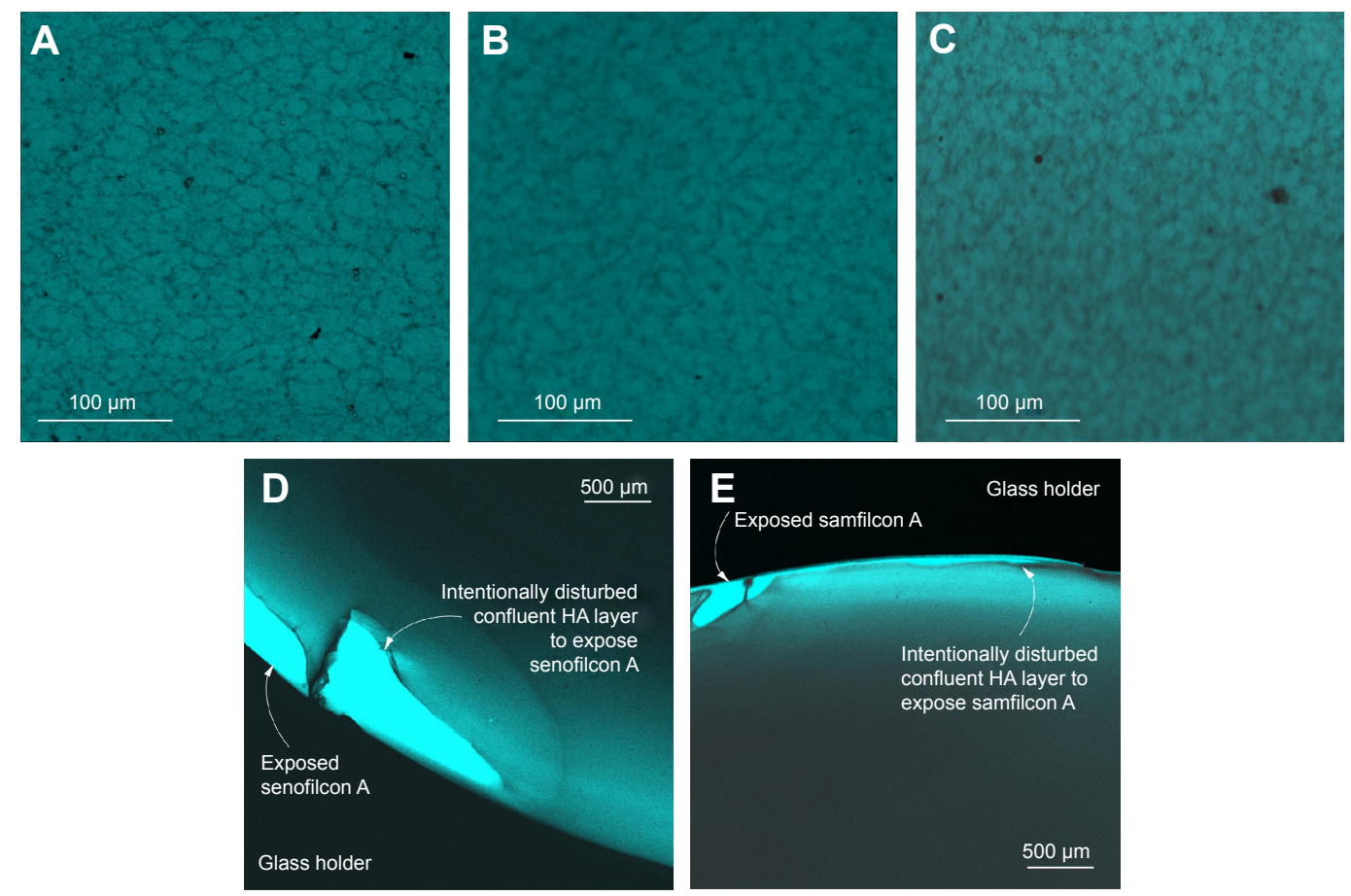

Figure 4 CLSM images.

Notes: Balafilcon $A(A)$, senofilcon $A(B)$, and samfilcon $A(C)$ incubated overnight with Biotrue MPS, then stained with safranin; senofilcon $A(D)$ and samfilcon $A(E)$ after scratching the sorbed HA layer with forceps. HA on balafilcon A could not be imaged by this method, because the stained lens did not sufficiently adhere to the glass slide. (A-C) Captured using a $20 \times$ microscope objective and $2 \times$ confocal magnification; (D-E) captured using a $4 \times$ microscope objective.

Abbreviations: CLSM, confocal laser-scanning microscopy; MPS, multipurpose solution; HA, hyaluronic acid. 
DIC imaging was used to resolve the fine details of the stained HA network, as well as to show its coverage over the lens surfaces (Figure 5). Lenses incubated with safranin solution but not exposed to HA or Biotrue MPS solutions served as control. The senofilcon A (Figure 5A) and samfilcon A (Figure 5D) control lens surfaces were smooth and clear. In contrast, the balafilcon A lens could not be imaged by DIC due to spectral interference. The refractive indices of HA-safranin complexes and stained polymer matrices were sufficiently different to visualize readily the confluent HA network present on senofilcon A (Figure 5, B and C) and samfilcon A (Figure 5, E and F) surfaces, respectively. The morphology, distribution, and density of the HA chains were similar to those observed in the CLSM images (Figure 4).

DIC has inherently lower resolution than either AFM or SEM, but is faster and does not require sample coating. DIC and CLSM imaging were used to demonstrate HA distribution over a large senofilcon A lens surface area. Individual confocal and DIC images captured sequentially across the sample with a $10 \times$ air objective were then combined into a large master image. While the HA pattern can be seen on individual images, the resulting master image was not convincingly stitched together correctly. Therefore, $10 \mu \mathrm{m}$-diameter beads were randomly distributed over the lens surface, and a second set of images labeled with beads was captured using the $10 \times$ microscopy objective. The superposition process was repeated using the beads to line up the frames, which resulted in a similar master image, corroborating the original procedure. Ten randomly chosen areas on both master images were examined using higher objective magnification $(20 \times)$ to verify HA coverage of the entire lens surface. The master images of HA sorbed on senofilcon A shown in Figures 6 (DIC) and 7 (CLSM) confirm uniform lens-surface coverage by the HA network. The DIC attachment on the Olympus CLSM allowed for recording of high-resolution images, as well as resolution of the fine details of the stained HA network. However, the lack of morphological detail of the network observed in Figure 6 was most likely due to the lost image resolution when beads rather than the HA were captured in focus.

Identification and characterization of interactions between HA and SiHy lens materials is beyond the scope of this study, but Ali and Byrne disclosed methods to increase the affinity of a hydrogel lens for HA. ${ }^{37}$ These authors incorporated into lens synthesis certain acrylate and methacrylate lens monomers to mimic amino acids from the HA-binding site
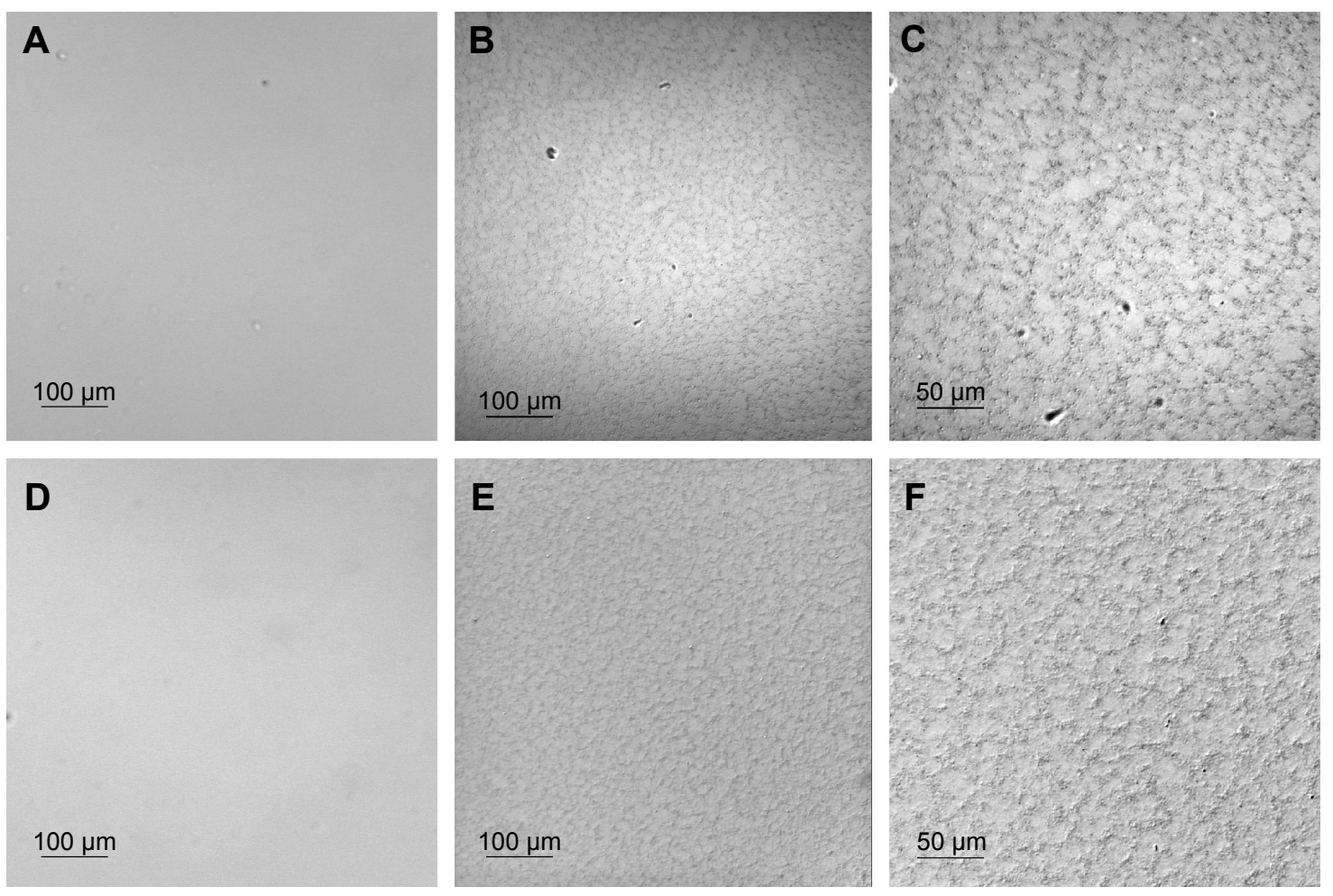

Figure 5 DIC images.

Notes: Senofilcon A (A) and samfilcon A (D) incubated with safranin only; senofilcon A (B, C) and samfilcon A (E, F) incubated overnight with Biotrue MPS, then stained with safranin. (A, B, D, E) Captured using a 10× microscope objective and $2 \times$ confocal magnification; (C, F) captured using a 20× objective and $2 \times$ confocal magnification. Abbreviations: DIC, differential interference contrast; MPS, multipurpose solution. 

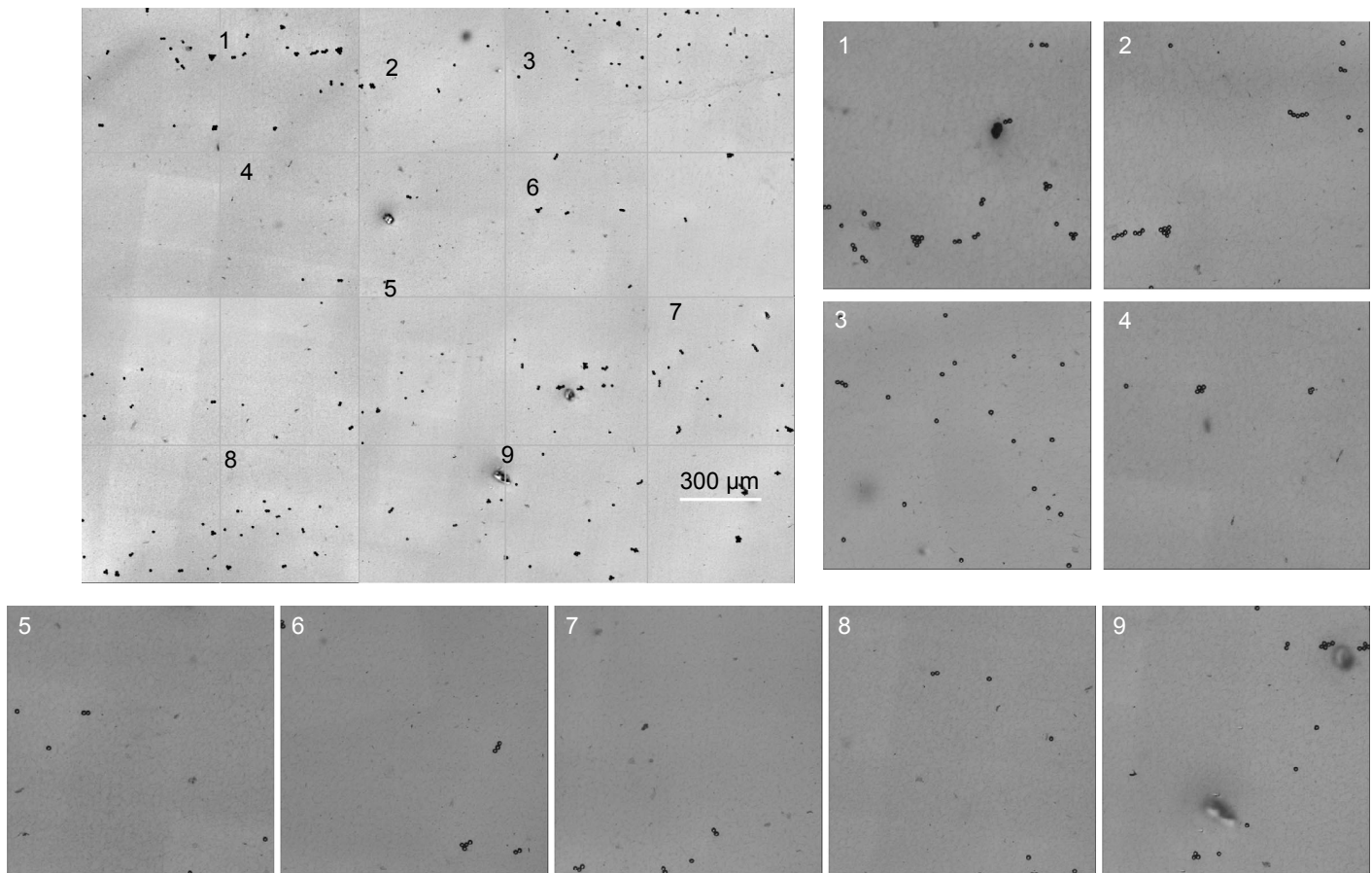

Figure 6 DIC image of HA distribution over a large senofilcon A lens area.

Notes: The master image of the HA network distributed over the entire lens area was prepared by stitching sequential images recorded using a 10x objective. Randomly chosen areas were examined using $20 \times$ magnification to visualize HA on the entire lens surface. The beads (fiducial marks) were used to identify fine details. Abbreviations: DIC, differential interference contrast; HA, hyaluronic acid.
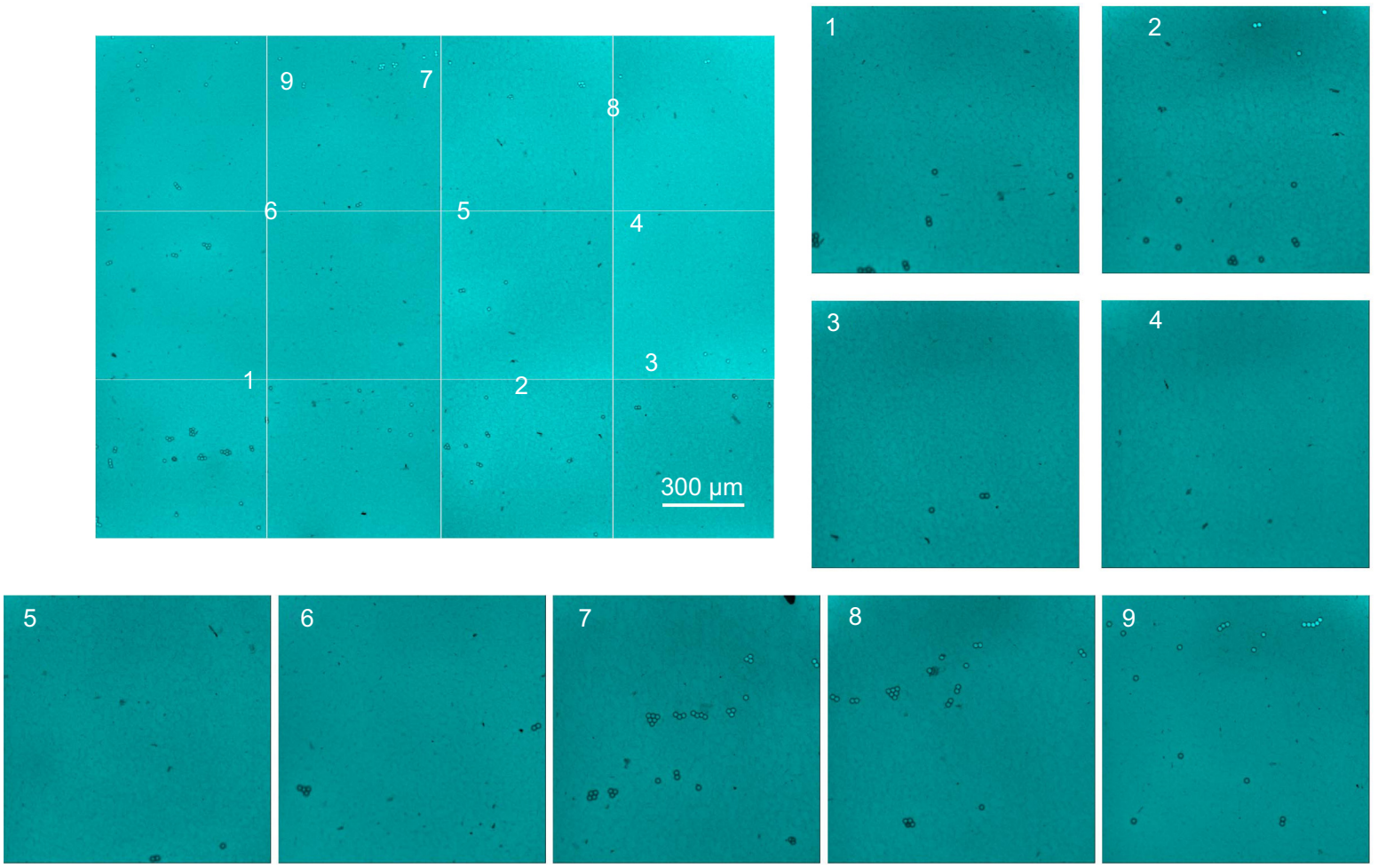

Figure 7 CLSM image of HA distribution over a large senofilcon A lens area.

Notes: The master image of the stained HA network distributed over the entire lens area was prepared by stitching sequential images recorded using a I0× objective. Randomly chosen areas were examined using $20 \times$ magnification to visualize the HA-network details. The beads (fiducial marks) were used to localize the image position on the master image.

Abbreviations: CLSM, confocal laser-scanning microscopy; HA, hyaluronic acid. 
of the natural CD44 receptor for HA. They further reported that among several molecules studied, PVP retarded HA diffusion from the lens bulk, due to noncovalent interaction. Therefore, similar noncovalent interaction between the PVP wetting agent present in samfilcon A and senofilcon A may promote HA attraction to these hydrogels.

The mechanism of the HA sorption on balafilcon A lenses may be more complicated. The relatively rougher topography of the hydrogel, ie, the pores, valleys, etc, may act as a physical binding site for HA. Additionally, the plasma treatment was not uniform over the entire lens surface, resulting in distinct hydrophilic and hydrophobic islands. Therefore, some functional group of the lens material may also interact with the HA, while others do not.

\section{Summary}

In this study, sorption of HA from Biotrue MPS to SiHy contact lens materials was investigated. Balafilcon A, senofilcon A, and samfilcon A were selected as model hydrogels. AFM and CLSM were used to characterize topography and RMS roughness of fresh (out of the package) SiHy materials, as well as to determine interaction with HA. AFM, CLSM, and DIC imaging confirmed the presence of a uniform HA network on the surfaces of all three SiHy lenses after incubation with HA solution or Biotrue MPS. While the inherent hydrophilicity of HA would lead one to expect that Biotrue MPS would closely associate with poly-HEMA-based contact lenses, this study suggests that it also readily associates with SiHy lenses. The unexpectedly high degree of nonspecific uptake of safranin into the lens bulk allowed for a simple method to visualize HA (and likely other high-MW biopolymers) on the lens surface. The technique should prove valuable in future studies of HA sorbed on contact lenses, and possibly HA sorbed on other biomedical polymers used in other applications.

\section{Disclosure}

The authors report no conflicts of interest in this work.

\section{References}

1. Epstein AA. Solution optimization and physical properties of healthy human tears. Contact Lens Spectr. 2010;25(4B):42-45.

2. Tonge S, Jones L, Goodall S, Tighe B. The ex vivo wettability of soft contact lenses. Curr Eye Res. 2001;23(1):51-59.

3. Ketelson HA, Meadows DM, McQueen N, Stone RP. Enhancing wettability with multi-purpose solutions. Rev Cornea Contact Lens. 2005;142(1 Suppl):44-47.

4. FDA 510(k) Summary K030092. COMPLETE ${ }^{\circledR}$ brand Multi-Purpose Solution. April 4, 2003. Available from: http://www.accessdata.fda.gov/ cdrh_docs/pdf3/k030092.pdf. Accessed May 25, 2016.
5. FDA $510(\mathrm{k})$ Summary K083812. REFRESH ${ }^{\circledR}$ OPTIVETM $^{\mathrm{TM}}$ Contacts Lubricating and Rewetting Drops. July 9, 2009. Available from: https:// www.accessdata.fda.gov/cdrh_docs/pdf8/K083812.pdf. Accessed May 25, 2016.

6. Scheuer CA, Fridman KM, Barniak VL, Burke SE, Venkatesh S. Retention of conditioning agent hyaluronan on hydrogel contact lenses. Cont Lens Anterior Eye. 2010;33 Suppl 1:S2-S6.

7. Scheuer CA, Doty K, Liranso T, Burke SE. Wetting agent retention and release from hydrogel and silicone hydrogel contact lenses. Invest Ophthalmol Vis Sci. 2011;52(14):6487.

8. FDA 510(k) Summary K083757. Bausch \& Lomb BPZO2 MultiPurpose Solution. November 18, 2009. Available from: http://www.accessdata. fda.gov/cdrh_docs/pdf8/k083757.pdf. Accessed May 25, 2016.

9. Fakhari A, Berkland C. Applications and emerging trends of hyaluronic acid in tissue engineering, as a dermal filler and in osteoarthritis treatment. Acta Biomater. 2013;9(7):7081-7092.

10. Lerner LE, Schwartz DM, Hwang DG, Howes EL, Stern R. Hyaluronan and CD44 in the human cornea and limbal conjunctiva. Exp Eye Res. 1998;67(4):481-484.

11. Brown MB, Jones SA. Hyaluronic acid: a unique topical vehicle for the localized delivery of drugs to the skin. J Eur Acad Dermatol Venereol. 2005;19(3):308-318.

12. Winter WT, Arnott S. Hyaluronic acid: the role of divalent cations in conformation and packing. J Mol Biol. 1977;117(3):761-784.

13. Rah M. A review of hyaluronan and its ophthalmic applications. Optometry. 2011;82(1):38-43.

14. Van Beek M, Jones L, Sheardown H. Hyaluronic acid containing hydrogels for the reduction of protein adsorption. Biomaterials. 2008;29(7): 780-789.

15. FDA 510(k) Summary K121201. Soft (Hydrophilic) Contact Lens, Oct 26, 2012. Available from: http://www.accessdata.fda.gov/cdrh_ docs/pdf12/K121201.pdf. Accessed May 25, 2016.

16. Liu XM, Harmon PS, Maziarz EP, Rah MJ, Merchea MM. Comparative studies of hyaluronan in marketed ophthalmic products. Optom Vis Sci. 2014;91(1):32-38.

17. Volpi N. Hyaluronic acid and chondroitin sulfate unsaturated disaccharides analysis by high-performance liquid chromatography and fluorimetric detection with dansylhydrazine. Anal Biochem. 2000; 277(1):19-24.

18. Mandair GS, Dehring KA, Roessler BJ, Morris MD. Detection of potential osteoarthritis biomarkers using surface enhanced Raman spectroscopy in the near-infrared. 2006. Available from: http://spie.org/ Publications/Proceedings/Paper/10.1117/12.644790. Accessed May 11, 2016.

19. Dehring KA, Mandair GS, Roessler BJ, Morris MD. Surface-enhanced Raman spectroscopy detection of hyaluronic acid: a potential biomarker for osteoarthritis. In: Kneipp K, Aroca R, Kneipp H, Wentrup-Byrne E, editors. New Approaches in Biomedical Spectroscopy. Washington: American Chemical Society; 2006:123-137.

20. Luo Y, Prestwich GD. Hyaluronic acid-N-hydroxysuccinimide: a useful intermediate for bioconjugation. Bioconjug Chem. 2001;12(6): 1085-1088.

21. Bennett R, Taher M. Restylane persistent for 23 months found during Mohs micrographic surgery: a source of confusion with hyaluronic acid surrounding basal cell carcinoma. Dermatol Surg. 2005;31(10): 1366-1369.

22. Tas J. The Alcian blue and combined Alcian blue-Safranin $O$ staining of glycosaminoglycans studied in a model system and in mast cells. Histochem J. 1977;9(2):205-230.

23. Whiteman P. The quantitative measurement of Alcian blue-glycosaminoglycan complexes. Biochem J. 1973;131(2):343-350.

24. Zanini A, Giannattasio G, Nussdorfer G, Margolis RK, Margolis RU, Meldolesi J. Molecular organization of prolactin granules. II. Characterization of glycosaminoglycans and glycoproteins of the bovine prolactin matrix. J Cell Biol. 1980;86(1):260-272. 
25. Fagnola M, Pagani MP, Maffioletti S, Tavazzi S, Papagni A. Hyaluronic acid in hydrophilic contact lenses: spectroscopic investigation of the content and release in solution. Cont Lens Anterior Eye. 2009;32(3): $108-112$.

26. FDA 510(k) Summary K122575. Bausch \& Lomb PureVision (balafilcon A) Visibility Tinted Contact Lens, December 20, 2012. Available from: https://www.accessdata.fda.gov/cdrh_docs/pdf12/ K122575.pdf. Accessed May 25, 2016.

27. FDA 510(k) Summary K131208. Bausch \& Lomb samfilcon A Contact Lens, September 11, 2013. Available from: https://www.accessdata.fda. gov/cdrh_docs/pdf13/K131208.pdf. Accessed May 25, 2016.

28. Wygladacz K, Hook D, Steffen R, Reindel W. Breaking the cycle of discomfort. Contact Lens Spectr. 2014;29(13):33-38.

29. FDA 510(k) Summary of Safety and Effectiveness K042275. VISTAKON $^{\circledR}$ (senofilcon A) Contact Lens, October 28, 2004. Available from: http://www.accessdata.fda.gov/cdrh_docs/pdf4/k042275. pdf. Accessed May 25, 2016.

30. Jones L, Subbaraman L, Rogers R, Dumbleton K. Surface treatment, wetting and modulus of silicone hydrogels. Optician. 2006;232(6067): 28-34.

31. Hutter JC, Green JA, Eydelman MB. Proposed silicone hydrogel contact lens grouping system for lens care product compatibility testing. Eye Contact Lens. 2012;38(6):358-362.

32. Reindel W, Cairns G, Merchea M. Assessment of patient and practitioner satisfaction with Biotrue ${ }^{\mathrm{TM}}$ multi-purpose solution for contact lenses. Cont Lens Anterior Eye. 2010;33 Suppl 1:S12-S17.

33. Bray BA. The role of hyaluronan in the pulmonary alveolus. $J$ Theor Biol. 2001;210(1):121-130.

34. González-Méijome JM, López-Alemany A, Almeida JB, Parafita MA, Refojo MF. Microscopic observation of unworn siloxane-hydrogel soft contact lenses by atomic force microscopy. J Biomed Mater Res B Appl Biomater. 2006;76(2):412-418.

35. Lira M, Santos L, Azeredo J, Yebra-Pimentel E, Oliveira ME. Comparative study of silicone-hydrogel contact lenses surfaces before and after wear using atomic force microscopy. J Biomed Mater Res B Appl Biomater. 2008;85(2):361-367.

36. Teichroeb JH, Forrest JA, Ngai V, Martin JW, Jones L, Medley J. Imaging protein deposits on contact lens materials. Optom Vis Sci. 2008;85(12): 1151-1164.

37. Ali M, Byrne ME. Controlled release of high molecular weight hyaluronic acid from molecularly imprinted hydrogel contact lenses. Pharm Res. 2009;26(3):714-726.
38. Weeks A, Morrison D, Alauzun JG, Brook MA, Jones L, Sheardown H. Photocrosslinkable hyaluronic acid as an internal wetting agent in model conventional and silicone hydrogel contact lenses. J Biomed Mater Res A. 2012;100(8):1972-1982.

39. Weeks A, Subbaraman LN, Jones L, Sheardown H. Physical entrapment of hyaluronic acid during synthesis results in extended release from model hydrogel and silicone hydrogel contact lens materials. Eye Contact Lens. 2013;39(2):179-185.

40. Bettuelli M, Trabattoni S, Fagnola M, Tavazzi S, Introzzi L, Farris S. Surface properties and wear performances of siloxane-hydrogel contact lenses. J Biomed Mater Res B Appl Biomater. 2013;101(8): $1585-1593$.

41. Tavazzi S, Tonveronachi M, Fagnola M, et al. Wear effects on microscopic morphology and hyaluronan uptake in siloxane-hydrogel contact lenses. J Biomed Mater Res B Appl Biomater. 2015;103(5): 1092-1098.

42. Schuster R, Schulenberg-Schell H. Applications of Fluorescence Detection in Liquid Chromatography: A New Approach to Lower Limits of Detection and Easy Spectral Analysis. Santa Clara (CA): Agilent Technologies; 2000.

43. Wygladacz K, Bakker E. Fluorescent microsphere fiber optic microsensor array for direct iodide detection at low picomolar concentrations. Analyst. 2007;132(3):268-272.

44. Blumenkrantz N. Microtest for mucopolysaccharides by means of toluidine blue: with special reference to hyaluronic acid. Clin Chem. 1957; 3(6):696-697.

45. Rosenberg L. Chemical basis for the histological use of safranin $\mathrm{O}$ in the study of articular cartilage. J Bone Joint Surg Am. 1971;53(1):69-82.

46. Koshkin V, Wang X, Scherer PE, Chan CB, Wheeler MB. Mitochondrial functional state in clonal pancreatic $\beta$-cells exposed to free fatty acids. J Biol Chem. 2003;278(22):19709-19715.

47. Weeks A, Boone A, Luensmann D, Jones L, Sheardown H. The effects of hyaluronic acid incorporated as a wetting agent on lysozyme denaturation in model contact lens materials. J Biomater Appl. 2013;28(3) 323-333.

48. Singh A, Li P, Beachley V, McDonnell P, Elisseeff JH. A hyaluronic acid-binding contact lens with enhanced water retention. Cont Lens Anterior Eye. 2015;38(2):79-84.
Clinical Ophthalmology

\section{Publish your work in this journal}

Clinical Ophthalmology is an international, peer-reviewed journal covering all subspecialties within ophthalmology. Key topics include: Optometry; Visual science; Pharmacology and drug therapy in eye diseases; Basic Sciences; Primary and Secondary eye care; Patient Safety and Quality of Care Improvements. This journal is indexed on Submit your manuscript here: http://www.dovepress.com/clinical-ophthalmology-journal

\section{Dovepress}

PubMed Central and CAS, and is the official journal of The Society of Clinical Ophthalmology (SCO). The manuscript management system is completely online and includes a very quick and fair peer-review system, which is all easy to use. Visit http://www.dovepress.com/ testimonials.php to read real quotes from published authors. 